\title{
Color-Tunable Hybrid White Organic Light-Emitting Diodes with Double Interlayers
}

\author{
Chunhong Gao*\#, Ziyang Xiong*, Fuxing Yu, Xingjuan Ma, Yue Zhang, \\ Xin Zeng, Zuhong Xiong
}

School of Physical Science and Technology, MOE Key Laboratory on Luminescence and Real-Time Analysis, Southwest University, Chongqing, China

Email: "gch0122@swu.edu.cn

How to cite this paper: Gao, C.H., Xiong, Z.Y., Yu, F.X., Ma, X.J., Zhang, Y., Zeng, X. and Xiong, Z.H. (2017) Color-Tunable Hybrid White Organic Light-Emitting Diodes with Double Interlayers. Optics and Photonics Journal, 7, 99-105.

https://doi.org/10.4236/opj.2017.78B014

Received: July 3, 2017

Accepted: August 7, 2017

Published: August 10, 2017

\begin{abstract}
An efficient color-tunable hybrid white organic light-emitting diode is demonstrated with double interlayers of 2,7-bis(carbazol-9-yl)-9,9-ditoylfluorene/2-(diphenylphosphoryl) spirofluorene (DMFL-CBP/SPPO1) inserted between blue fluorescent and yellow phosphorescent-emitting layers, and exhibits Commission Internationale de l'Eclairage (CIE1931) ranging from warm white $(0.4368,0.4497)$ to cool white $(0.2781,0.2896)$ with driving current density from 0.2 to $40 \mathrm{~mA} / \mathrm{cm}^{2}$. The recombination of singlet and the triplet excitons in blue fluorescent-emitting layer and yellow phosphorescent-emitting layer, respectively, can be modulated by both the thickness of these double interlayers and the applied current densities.
\end{abstract}

\section{Keywords}

Hybrid White Organic Light-Emitting Diode, Color-Tunable, Singlet Exciton, Triplet Exciton, Phosphor

\section{Introduction}

Since the first practical sandwiched organic light-emitting diode (OLED) was reported by C.W. Tang in 1980s [1], thirty years have been passed with more and more attention paid in this field due to low cast, flexibility, area source, and high response [2] [3] [4]. Hybrid white organic light-emitting diodes (HWOLEDs), which posses the advantage of both the long stability of the blue fluorescent-emitting layer based OLEDs and the high electroluminescent efficiency of yellow phosphorescent-emitting layer based OLEDs, have been considered as a potential light source for lighting and display [5].

*These authors contributed equally in this work. 
To prevent the singlet energy transfer from the blue fluorescent emitting layer to the complement phosphorescent emitting layer and to blocking the triplet excitons in the complement phosphorescent emitting layer, interlayer with high singlet and triplet energy should be inserted between the two layers. [6] Kinds of structures have been created to get high efficiency and long stability HWOLEDs. In 2006, Sun et al. proposed HWOLEs with the blue fluorophor-single interlayer-complementary phosphor-single interlayer-blue fluorophor (F-SI-P-SI-F) structure, which can harvest both singlet and triplet excitions and exhibit a highly efficiency of $23.8 \mathrm{~lm} / \mathrm{W}$ at $500 \mathrm{~cd} / \mathrm{m}^{2}$ [7]. To simplify the device structure, the architectures of HWOLEDs with the complementary phosphor-single interlayer-blue fluorophor (P-SI-F) structure [8] [11], and the complementary phosphor-mixed single interlayer-blue fluorophor (P-MSI-F) structure [14] [15] are developed to manipulate the charges and excitions in the HWOLEDs. Normally, hole transporting material (N,N-dicarbazoyl-3,5-benzene, $\mathrm{mCP}$ ) [8], electron transporting material (2,9-dimethyl-4,7-diphenyl-1,10-phenanthroline, BCP) [9], and ambipolar transporting material (4,4'-bis(carbazol-9-yl)biphenyl, CBP) [10] [12] [13] are used as the single interlayers. The mixed single interlayer is formed by co-evaporating hole transporting material (or ambipolar transporting material) and electron transporting material to modulate the shape of the color spectra [16] [17]. However, seldom double interlayers are reported in the HWOLEDs.

In this work, double interlayers of 2,7-bis(carbazol-9-yl)-9,9-ditoylfluorene/2(diphenylphosphoryl) spirofluorene (DMFL-CBP/SPPO1) is inserted between the blue fluorescent emitting layer and yellow phosphorescent emitting layer to manipulate the exciton generating zone. An efficient HWOLED is realized with a large chrominance span from CIE $(0.4368,0.4497)$ to CIE $(0.2781,0.2896)$.

\section{Experimental Section}

After the $110 \mathrm{~nm}$ indium-tin-oxide (ITO) film coated glass substrate with a sheet resistance of $15 \Omega / \square$ is treated by UV-ozone for $15 \mathrm{~min}$, layers of molybdenum trioxide $\left(\mathrm{MoO}_{3}\right), \mathrm{N}, \mathrm{N}^{\prime}$-di(1-naphthyl)-N,N'-diphenyl-(1,1'-biphenyl)-4,4'-diamine (NPB), 2-methyl-9,10-bis(naphthalen-2-yl)anthracene: 4,4'-bis(9-ethyl-3carbazovinylene)-1,1'-biphenyl (MADN: BCzVBi), DMFL-CBP/SPPO1, 2',2"(1,3,5-benzinetriyl)-Tris(1-phenyl-1-H-benzimidazole):Iridium(III)bis(4-phenyl thieno[3,2-c]pyridinato-N,C2')acetylacetonate (TPBi: PO-01), bathophenanthroline (Bphen), tris-8-hydroxyquinoline aluminium (Alq), lithium quinolate/ aluminum ( $\mathrm{Liq} / \mathrm{Al})$, which are used as hole injecting layer, hole transporting layer, blue fluorescent emitting layer, double interlayers, yellow phosphorescent emitting layer, hole blocking layer, electon transporting layer, and bilayer cathode, respectively, are subsequently thermally evaporated under a base pressure of $2 \times 10^{-6}$ Torr. The device structures of the HWOLEDs consist of ITO/ $\mathrm{MoO}_{3}(10 \mathrm{~nm}) / \mathrm{NPB}(75 \mathrm{~nm}) / \mathrm{MADN}$ : BCzVBi $7 \mathrm{wt} \%$ (20 nm)/DMFL-CBP (X)/ SPPO1 (Y)/TPBi: PO-0.13 wt\% $(20 \mathrm{~nm}) /$ Bphen $(10 \mathrm{~nm}) /$ Alq $(20 \mathrm{~nm}) / \mathrm{Liq}(2$ 
$\mathrm{nm}) / \mathrm{Al}(120 \mathrm{~nm})$, where the sum of " $\mathrm{X}$ " and " $\mathrm{Y}$ " is 5 , and " $\mathrm{X}$ " stands for $3 \mathrm{~nm}$, $2.5 \mathrm{~nm}, 2 \mathrm{~nm}$ in Device A, Device B, and Device C, respectively. The schematic diagram and the energy level graph of device strucures are shown in Figure 1(a) and Figure 1(b) [18] [19] [20].

The oscillating quartz thickness monitor was used to measure the deposition rate and thickness of the layers in situ. The Photo Research Spectra Scan PR655 photometer and Keithley 2400 Source Meter were connected by software to measure the current-voltage curves, luminance, the electroluminescent (EL) spectra, CIE coordinates of the HWOLEDs. The active area of each HWOLED is $0.1 \mathrm{~cm}^{2}$.

\section{Results and Discussion}

Figure 2 shows the EL performance of the three HWOLEDs with different double interlayers of DMFL-CBP (3 nm)/SPPO1 (2 nm), DMFL-CBP (2.5 nm)/ SPPO1 (2.5 nm), DMFL-CBP (2 nm)/SPPO1 (3 nm) for Devices A, B and C, respectively. In current density-voltage-luminance curves in Figure 2(a), it can be found the thicker the DMFL-CBP, the lower the current density and the larger the luminance at each applied voltage. In Figure 2(b) and Figure 2(c), current efficiency and power efficiency increased with the increasing DMFL-CBP thickness at each applied current density, and shows the highest current efficiency and power efficiency of $36.5 \mathrm{~cd} / \mathrm{A}$ and $30.1 \mathrm{~lm} / \mathrm{W}$ driving at $0.2 \mathrm{~mA} / \mathrm{m}^{2}$ in Device A. And Devices B (C) shows maximum current efficiency and power efficiency of $27 \mathrm{~cd} / \mathrm{A}(24.5 \mathrm{~cd} / \mathrm{A})$ and $22.7 \mathrm{~lm} / \mathrm{W}(20.1 \mathrm{~lm} / \mathrm{W})$, respectively. These are attributed to that the exciton generation interface should be located at the interface of DMFL-CBP/SPPO1 owing to the high lowest unoccupied molecular orbital (LUMO) of DMFL-CBP $(-1.29 \mathrm{eV})$ and the low highest occupied molecular orbital (HOMO) of SPPO1 $(-6.5 \mathrm{eV})$ which can block electrons and holes at this interface, respectively.
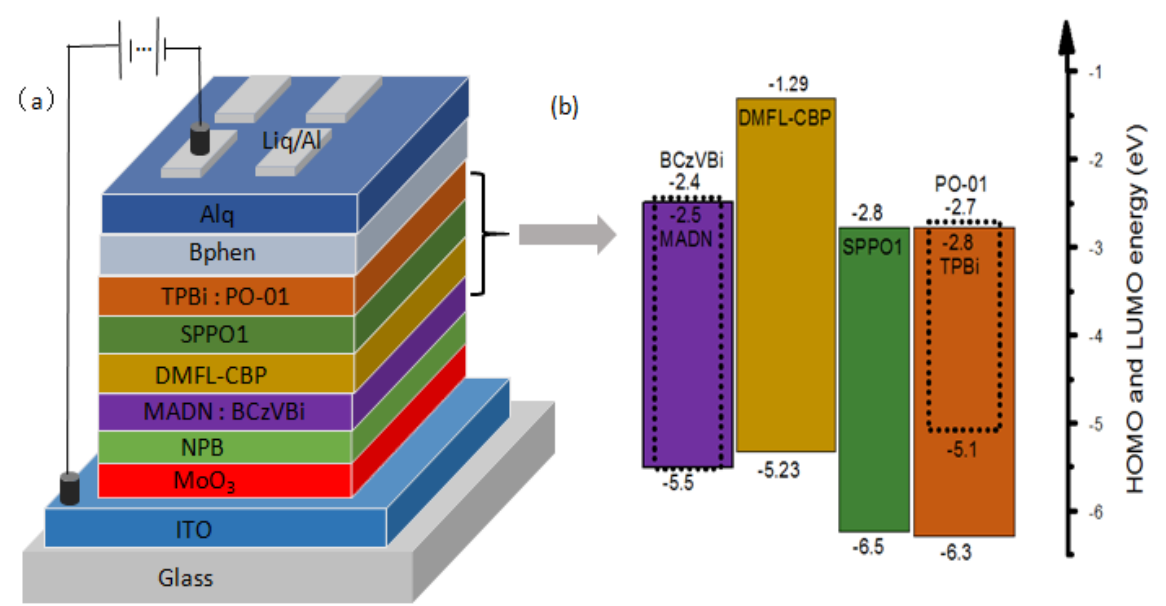

Figure 1. The schematic diagram (a) and energy level graph of the HWOLEDs (Devices A, B and C). 

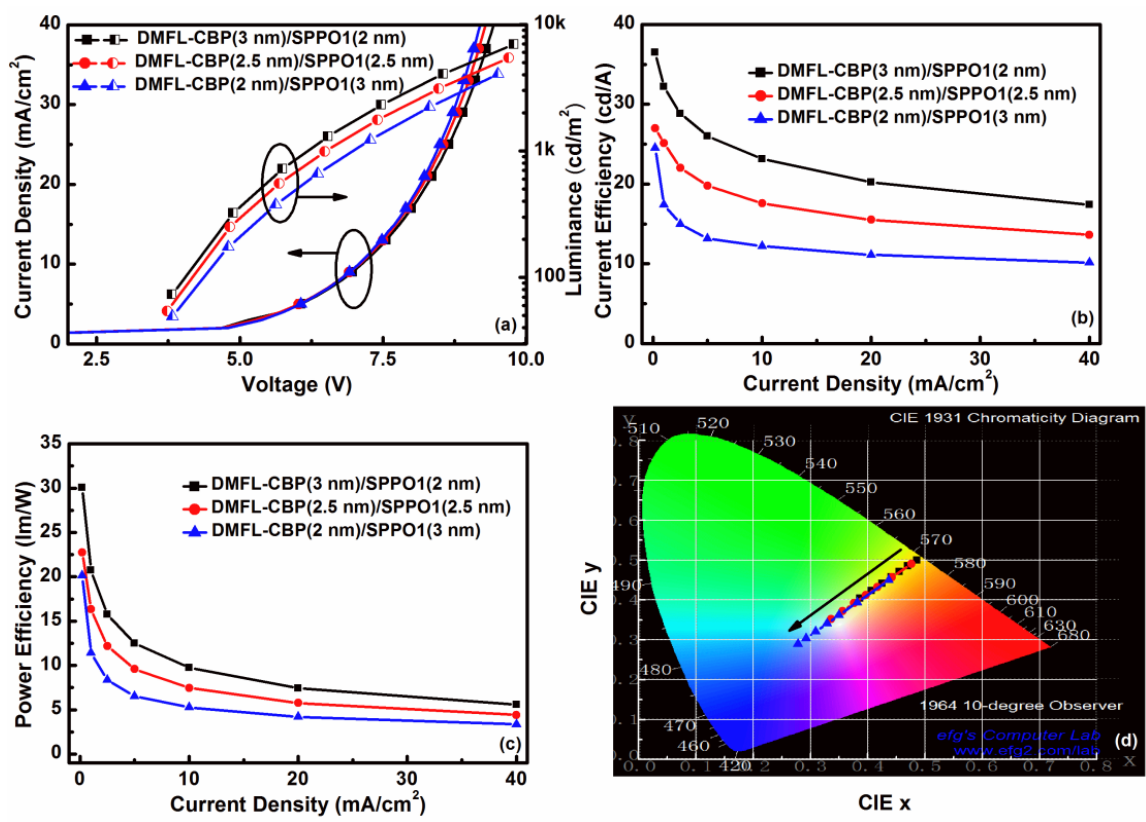

Figure 2. (a) The current density-voltage-luminance relationship; (b) The current efficiency-current density relationship; (c) The power efficiency-current density relationship of Devices A, B, and C with double intelayers of DMFL-CBP (3 nm)/SPPO1 (2 nm), DMFL-CBP (2.5 nm)/SPPO1 $(2.5 \mathrm{~nm})$, DMFL-CBP $(2 \mathrm{~nm}) / \mathrm{SPPO} 1(3 \mathrm{~nm})$, respectively; (d) The CIE $(x, y)$ coordinates of the three devices, in which the square, circle, and triangle symbol for Devices A, B, and C, respectively. And the direction of the arrow represents the increase trend of applied current density from 0.2 to $1,2.5,5,10,20,40 \mathrm{~mA} / \mathrm{cm}^{2}$.

When the thickness of DMFL-CBP increased, the exciton generation interface shift closer to the yellow EML, and thus leading to more exciton diffused into the yellow EML. Moreover, the efficiency of OLEDs with PO-01 as emitter is larger than that of OLEDs with $\mathrm{BCzVBi}$ as emitter. So, higher efficiency are achieved in the HWOLEDs with thicker DMFL-CBP. In Figure 2(d), when the driving current density increase from $0.2 \mathrm{~mA} / \mathrm{cm}^{2}$ to $40 \mathrm{~mA} / \mathrm{cm}^{2}$, the CIE coordinates of Device A with DMFL-CBP $(3 \mathrm{~nm}) / \mathrm{SPPO} 1(2 \mathrm{~nm})$ changes from yellow $(0.4858$, $0.5000)$ to warm white $(0.3865,0.4038)$. And the CIE coordinates of the Device $B$ with DMFL-CBP $(2.5 \mathrm{~nm}) / \mathrm{SPPO} 1(2.5 \mathrm{~nm})$ changes from yellow $(0.4766$, $0.4909)$, across warm white $(0.4171,0.4324)$ to white $(0.336,0.3524)$. For the Device $C$ with DMFL-CBP $(2 \mathrm{~nm}) / \mathrm{SPPO} 1(3 \mathrm{~nm})$, the CIE coordinates ranges from warm white $(0.4368,0.4497)$, across white $(0.3289,0.3408)$ to cool white $(0.2781$, 0.2896). The color-tunable HWOLEDs may satisfy people's different demands in different situations.

To investigate the physical mechanism in these HWOLEDs, the normalized EL spectra driving at $0.2,1,2.5,5,10,20,40 \mathrm{~mA} / \mathrm{cm}^{2}$ are studied and exhibited in Figure 3 with the yellow emission peak intensity (located at $560 \mathrm{~nm}$ ) as the reference intensity. The proportion of blue emission part in the normalized EL spectrum increased with the increasing current density in each devices. This may be caused by that more singlets are captured by the blue fluorescent emitter 

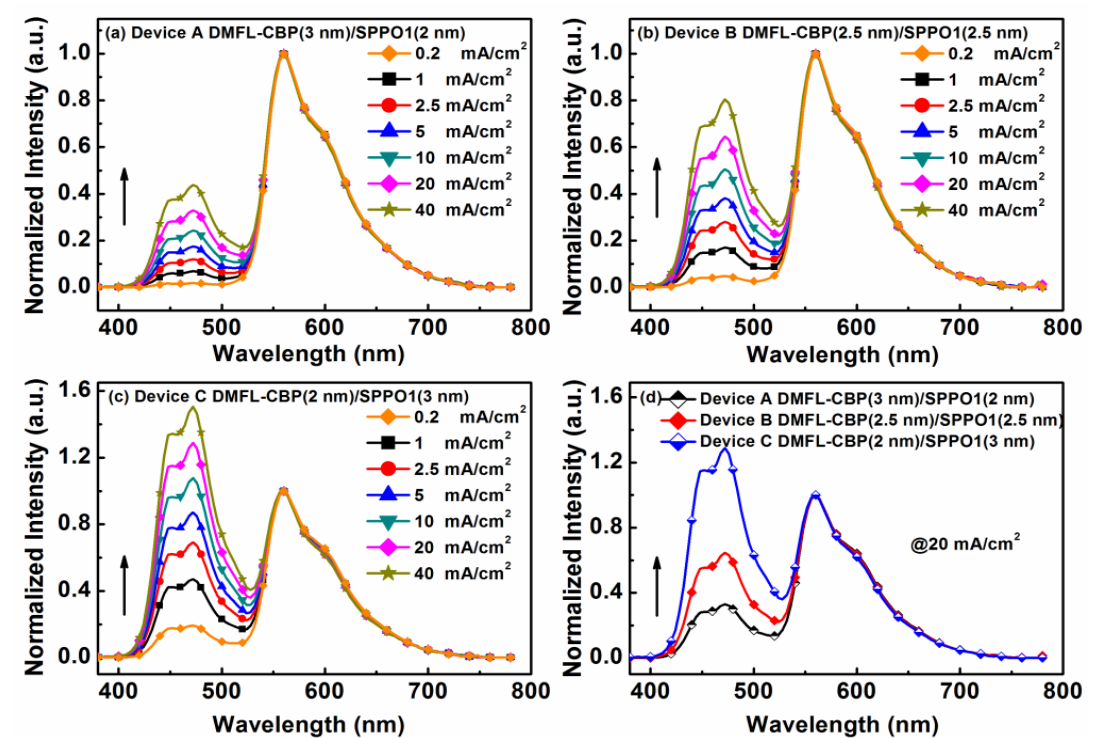

Figure 3. (a) The normalized EL spectra of Device A; (b) The normalized EL spectra of Device B; (c) The normalized EL spectra of Device C driving at current densities of 0.2, 1 , $2.5,5,10,20,40 \mathrm{~mA} / \mathrm{cm}^{2}$; (d) The EL spectra of Devices A, B, and C driving at 20 $\mathrm{mA} / \mathrm{cm}^{2}$.

$\mathrm{BCzVBi}$, due to more electrons injected in to the blue EML across the DMFLCBP layer with the increased current density. When the current density is fixed such as $20 \mathrm{~mA} / \mathrm{cm}^{2}$, the proportion of blue emission part goes up with the thickness of the DMFL-CBP decreasing, because the exciton generation interface DMFL-CBP/SPP0O1 is closer to the blue fluorescent EML and thus more singlet emitting blue light via $\mathrm{BCzVBi}$.

\section{Conclusion}

A hybrid white light-emitting diode with widespread color CIE coordinates ranging from warm white $(0.4368,0.4497)$, across white $(0.3289,0.3408)$ to cool white $(0.2781,0.2896)$ is demonstrated by incorporating double interlayers of DMFL-CBP/SPPO1 between the blue fluorescent emitting layer and the yellow phosphorescent emitting layer. The color CIE coordinates can be tuned by both the thickness ratio of DMFL-CBP to SPPO1 and the driving current density, due to relative change of the exciton generation interface and the electron injecting ability into blue fluorescent emitting layer.

\section{Acknowledgements}

We acknowledge financial support from the Natural Science Foundation of China (Grant Nos. 61404108), and Fundamental Research Funds for the Central Universities (Grant Nos. XDJK2017D140).

\section{Conflict}

The authors declare no competing financial interest. 


\section{References}

[1] Tang, C.W. and VanSlyke, S.A. (1987) Organic Electroluminescent Diodes. Applied Physics Letters, 51, 913-915. https://doi.org/10.1063/1.98799

[2] Reineke, S., Lindner, F., Schwartz, G., Seidler, N., Walzer, K., Lussem, B., Leo, K. (2009) White Organic Light-Emitting Diodes with Fluorescent Tube Efficiency. Nature, 459, 234-U116. https://doi.org/10.1038/nature08003

[3] Uoyama, H., Goushi, K., Shizu, K., Nomura, H. and Adachi, C. (2012) Highly Efficient Organic Light-Emitting Diodes from Delayed Fluorescence. Nature, 492, 234 238. https://doi.org/10.1038/nature 11687

[4] Williams, E.L., Haabisto, K., Li, J. and Jabbour, G.E. (2007) Excimer-Based White Phosphorescent Organic Light-Emitting Diodes with Nearly $100 \%$ Internal Quantum Efficiency. Advanced Materials, 19, 197-202.

https://doi.org/10.1002/adma.200602174

[5] Lee, B.M., Yoo, S.I., Kang, J.S., Yoon, J.A., Kim, W.Y. and Mascher, P. (2016) Hybrid Blue Organic Light Emittin Diodes with Fluorescentand Phosphorescent Emitters Alongwith an Interlayer. Science of Advanced Materials, 8, 301-306. https://doi.org/10.1166/sam.2016.2483

[6] Gao, C.H., Shi, X.B., Zhou, D.Y., Zhang, L., Wang, Z.K. and Liao, L.S. (2013) Highly Efficient White Organic Light-Emitting Diodes with Controllable Excitons Behavior by a Mixed Interlayer between Fluorescence Blue and Phosphorescence YellowEmitting Layers. International Journal of Photoenergy, 2013, 1-7. https://doi.org/10.1155/2013/831765

[7] Sun, Y., Giebink, N.C., Kanno, H., Ma, B., Thompson, M.E. and Forrest, S.R. (2006) Management of Singlet and Triplet Excitons for Efficient White Organic LightEmitting Devices. Nature, 404, 908-912. https://doi.org/10.1038/nature04645

[8] Leem, D.S., Kim, J.W., Jung, S.O., Kim, S.O., Kim, S.H., Kim, K.Y., Kim, Y.H., Kwon, S.K. and Kim, J.J. (2010) Efficient and Colour-Stable Hybrid White Organic Light-Emitting Diodes Utilizing Electron-Hole Balanced Spacers. Journal of Physics D: Applied Physics, 43, 405102. https://doi.org/10.1088/0022-3727/43/40/405102

[9] Zamani, S.H., Luo, Y. and Aziz, H. (2011) Luminescence Degradation in Phosphorescent Organic Light-Emitting Devices by Hole Space Charges. Journal of Applied Physics, 109, 044501-044501-6. https://doi.org/10.1063/1.3549128

[10] Chen P., Xue, Q., Xie, W., Duan, Y., Xie, G., Zhao, Y., Hou, J.Y., Liu, S.Y., Zhang, L.Y. and Li, B. (2008) Color-Stable and Efficient Stacked White Organic LightEmitting Devices Comprising Blue Fluorescent and Orange Phosphorescent Emissive Units. Applied Physics Letters, 93, 153508-153508-3. https://doi.org/10.1063/1.2998598

[11] Li, X.L., Ouyang, X.H., Liu, M., Ge, Z.Y., Peng, J.B., Gao, Y. and Su, S.J. (2015) Highly Efficient Single- and Multi-Emission-Layer Fluorescent/Phosphorescent Hybrid White Organic Light-Emitting Diodes with 20\% External Quantum Efficiency. Journal of Materials Chemistry C, 3, 9233-9239. https://doi.org/10.1039/C5TC02050F

[12] Yang, S.H., Shih, P.J., Wu, W.J. and Huang, Y.H. (2013) Color-Tunable and StableEfficiency White Organic Light-Emitting Diode Fabricated with FluorescentPhosphorescent Emission Layers. Journal of Luminescence, 142, 86-91. https://doi.org/10.1016/j.jlumin.2013.03.060

[13] Zheng, T. and Choy, W.C.H. (2010) Red Organic Light Emitting Devices with Reduced Efficiency Roll-Off Behavior by Using Hybrid Fluorescent/Phosphorescent 
Emission Structure. Thin Solid Films, 519, 872-875. https://doi.org/10.1016/j.tsf.2010.09.010

[14] Yook, K.S., Jeon, S.O., Joo, C.W. and Lee, J.Y. (2008) High Efficiency, Color Stability, and Stable Efficiency Roll Off in Three Color Hybrid White Organic Light Emitting Diodes. Applied Physics Letters, 93, 073302. https://doi.org/10.1063/1.2969821

[15] Schwartz, G., Ke, T.H., Wu, C.C., Walzer, K. and Leo, K. (2008) Balanced Ambipolar Charge Carrier Mobility in Mixed Layers for Application in Hybrid White Organic Light-Emitting Diodes. Applied Physics Letters, 93, 073304. https://doi.org/10.1063/1.2973151

[16] Mesta, M., Carvelli, M., Vries, R.J., Eersel, H., Holst, J.J.M., Schober, M., Furno, M., Lüssem, B., Leo, K., Loebl, P., Coehoorn, R. and Bobber, P.A. (2013) MolecularScale Simulation of Electroluminescence in a Multilayer White Organic LightEmitting Diode. Nature Materials, 12, 652-658. https://doi.org/10.1038/nmat3622

[17] Schwartz, G., Reineke, S., Walzer, K. and Leo, K. (2008) Reduced Efficiency Roll-Off in High-Efficiency Hybrid White Organic Light-Emitting Diodes. Applied Physics Letters, 92, 053311. https://doi.org/10.1063/1.2836772

[18] Wu, X.M., Zhang, X., Bai, J.J., Hua, Y.L., Xin, L.W., Yu, Q.Q. and Yin, S.G. (2015) Utilization of a Composite Hole Transporting Layer and Novel Homogeneous Double Emitting Layers for Performance Improvement and Low Efficiency Roll-Off in Organic Light-Emitting Diodes. Displays, 38, 62-67. https://doi.org/10.1016/j.displa.2015.03.004

[19] Choi, A.Y., Yamaguchi, T. and Han, C.H. (2012) A Photochemical Investigation into Operational Degradation of Arylamines in Organic Light-Emitting Diodes. Research on Chemical Intermediates, 39, 1571-1579. https://doi.org/10.1007/s11164-012-0613-y

[20] Lee, J., Sung, W.J., Joo, C.W., Cho, H., Cho, N., Lee, G.W., Hwang, D.H. and Lee, J.I. (2016) Simplified Bilayer White Phosphorescent Organic Light-Emitting Diodes. ETRI Journal, 38, 260-264. https://doi.org/10.4218/etrij.16.2515.0011

\section{Submit or recommend next manuscript to SCIRP and we will provide best service for you:}

Accepting pre-submission inquiries through Email, Facebook, LinkedIn, Twitter, etc. A wide selection of journals (inclusive of 9 subjects, more than 200 journals)

Providing 24-hour high-quality service

User-friendly online submission system

Fair and swift peer-review system

Efficient typesetting and proofreading procedure

Display of the result of downloads and visits, as well as the number of cited articles

Maximum dissemination of your research work

Submit your manuscript at: http://papersubmission.scirp.org/

Or contact opj@scirp.org 